\title{
Changes in the Distribution of GAP-43 During the Development of Neuronal Polarity
}

\author{
Kimberly Goslin,, ${ }^{1, a}$ David J. Schreyer, ${ }^{2}$ J. H. Pate Skene, ${ }^{2}$ and Gary Banker ${ }^{1 . a}$ \\ ${ }^{1}$ Department of Anatomy, Cell Biology, and Neurobiology, Albany Medical College, Albany, New York 12208, and \\ aDepartment of Neurobiology, Stanford University School of Medicine, Stanford, California 94305
}

GAP-43, a neuron specific growth-associated protein, is selectively distributed to the axonal domain in developing neurons; it is absent from dendrites and their growth cones. Using immunofluorescence microscopy, we have further examined the distribution of GAP-43 during the development of hippocampal neurons in culture, in order to determine when this polarized distribution arises. Cultured hippocampal neurons initially extend several short processes which have the potential to become either axons or dendrites. At this stage, before the morphological expression of polarity, GAP-43 is concentrated in the growth cones of these processes but is distributed more or less equally among them. Polarity becomes established when one of these processes elongates to become the axon. At the earliest stage when the emerging axon can be identified, GAP-43 is preferentially concentrated in its growth cone. During the next few days, as the remaining processes take on dendritic properties, they lose their residual GAP-43 immunoreactivity. Throughout development, GAP-43 remains highly concentrated in the axonal growth cone, but the concentration of GAP-43 in the axon shaft increases, beginning near the growth cone and progressing proximally until GAP-43 is uniformly distributed along the entire axon. At all stages of development, GAP43 is also concentrated in the region of the Golgi apparatus.

These results suggest that the selective sorting of at least one membrane protein into the axon coincides with the morphological expression of polarity. These results also raise the possibility that GAP-43 may play an important role in the early phases of axonal outgrowth, by which the functional polarity of neurons is established.

The development of distinct axonal and dendritic domains provides the basis for the functional polarization of neurons. Cell cultures prepared from the hippocampal region of the cerebral cortex have proven to be a useful system for examining the cellular mechanisms underlying the development of neuronal polarity. Hippocampal neurons in culture develop distinct axons

Received July 11, 1989; revised Aug. 23, 1989; accepted Aug. 29, 1989.

We wish to thank Dr. L. Binder for the gift of antibodies against MAP2 and Dr. P. DeCanilli for the gift of antibodies against Synapsin 1 and for his critical reading of the manuscript. This work was supported by NIH Grants NS17112 (to G.B.) and NS20178 (to J.H.P.S.)

Correspondence should be addressed to Dr. Gary Banker, Department of Neuroscience, University of Virginia School of Medicine, Box 230, Medical Center Charlottesville, VA 22908.

a Present address: Department of Neuroscience, Iniversity of Virginia School of Medicine, Charlottesville, VA.

Copyright (C) 1990 Society for Neuroscience $0270-6474 / 90 / 100588-15 \$ 02.00 / 0$ and dendrites, which, like their counterparts in situ, differ from one another in light and electron microscopic morphology (Bartlett and Banker, 1984a, b), in the macromolecular composition of their cytoskeletal and membranous constituents (Shaw et al., 1985; Caceres et al., 1986; Matus et al., 1986; Goslin et al., 1988), and in synaptic polarity (Bartlett and Banker, 1984b). In such cultures, where the differential outgrowth of axons and dendrites can be readily observed, the sequence of events leading to the development of a polarized form has been well documented (Dotti et al., 1988). Early in development hippocampal neurons extend several, short processes, but show no indication of polarity. These processes may become either axons or dendrites (Dotti and Banker, 1987; Goslin and Banker, 1989). Polarity is first expressed when, rather abruptly, one of these processes begins to grow rapidly and acquires axonal characteristics. The remaining processes elongate somewhat later and take on the properties of dendrites.

We have recently found that the neuron-specific protein GAP43 (also termed B-50, F1, P57, or pp46) is present in axons and axonal growth cones but is not detectable in growing dendrites or dendritic growth cones of hippocampal neurons in culture (Goslin et al., 1988). Previous work has shown that the expression of GAP-43 is closely correlated with neurite growth, both during development and regeneration (Skene and Willard, 198 lac; Benowitz and Lewis, 1983; Redshaw and Bixby, 1984; Jacobson et al., 1986; Benowitz and Routtenberg, 1987; Skene, 1989). GAP-43 is a prominent protein component of growth cones, the motile tips of elongating neurites, comprising approximately $1 \%$ of the total protein of growth cone membranes (Meiri et al., 1986, 1988; Skene et al., 1986). In view of the implied role of GAP-43 in regulating neurite growth, its selective distribution in developing neurons raises the possibility that, by mediating the differential outgrowth of axons, it may be important in the establishment of neuronal polarity. Studies of GAP-43 at early stages of neuronal development are necessary to determine if its expression and localization are consistent with such a possibility. Such studies may also provide information about the selection of different membrane proteins for transport into growing axons and dendrites. The selective sorting of membrane components is of fundamental importance for the development and maintenance of polarity in non-neuronal cells (Simons and Fuller, 1985; Griffiths and Simons, 1986), and is likely to be equally important in the development of neuronal polarity.

In the present study, we used immunofluorescence microscopy to investigate the localization of GAP-43 during the development of hippocampal neurons in culture, where changes in its distribution can be followed at the single-cell level. Our 
results reveal that in unpolarized neurons, GAP-43 is concentrated more-or-less equally in the growth cones of all of the processes. As the axon begins to elongate, GAP-43 becomes highly concentrated in its growth cone. Thus, the selective sorting of GAP-43 to the axon becomes apparent coincidently with the morphological expression of polarity. Because its selective distribution arises at this early stage, GAP-43 could be involved in the mechanisms which underlie the establishment of neuronal polarity.

\section{Materials and Methods}

Preparation of hippocampal cell cultures. Cell cultures were prepared from hippocampi of 18-d-old fetal rats, as described previously (Banker and Cowan, 1977; Bartlett and Banker, 1984a). In brief, cell suspensions were obtained by trypsin treatment $\left(0.25 \%\right.$ for $15 \mathrm{~min}$ at $\left.37^{\circ} \mathrm{C}\right)$ and dissociation by repeated passage through a fire-polished Pasteur pipet. The cells, at a density of about $6000 / \mathrm{cm}^{2}$, were plated onto poly-Llysine-treated glass coverslips in MEM containing $10 \%$ horse serum. After $3-4 \mathrm{hr}$, when the cells had attached, the coverslips were transferred to dishes containing an astroglial monolayer (Booher and Sensenbrenner, 1972), so that they faced, but did not contact, the glia. Previous work has shown that this coculture technique enhances long-term neuronal survival (Banker, 1980). The cells were maintained in MEM containing the $\mathrm{N}_{2}$ supplements of Bottenstein and Sato (1979) plus ovalbumin $(0.1 \%)$ and pyruvate $(0.1 \mathrm{~mm})$

Immunoblots. Cultures of hippocampal neurons were solubilized by the addition of $2 \%$ SDS, $5 \% 2$-mercaptoethanol, and $65 \mathrm{~mm}$ Tris- $\mathrm{HCl}$ ( $\mathrm{pH}$ 6.8); hippocampi from $3 \mathrm{~d}$ postnatal rats were homogenized in this same solution. The samples were then boiled for $2 \mathrm{~min}$ and stored at $-80^{\circ} \mathrm{C}$. After thawing, samples containing $40-140 \mu \mathrm{g}$ protein were loaded onto a discontinuous, $12 \%$ polyacrylamide gel containing SDS and electrophoresed according to the method of Laemmli (1970). The gel containing resolved proteins was immediately electroblotted onto nitrocellulose using a semidry transfer apparatus and blotting buffer consisting of $40 \mathrm{~mm}$ 3-[dimethyl(hydroxymethyl)-methylamino]-2-hydroxypropane sulfonic acid and $20 \%$ methanol (pH 9.5). The nitrocellulose was immersed in a blocking solution containing $2 \%$ horse serum and $0.5 \%$ BSA in PBS for $30 \mathrm{~min}$. It was then immunoprobed with anti-GAP-43 monoclonal antibody 9-1E12 (ascites fluid diluted 1: $2000)$ or rabbit anti-GAP-43 antiserum (1:100) for 1 hr. The pattern of antibody binding was developed using an avidin-biotin-peroxidase kit (Vectastain ABC Kit, Vector Laboratories, Burlingame, CA) according to the manufacturer's instructions, except that reagents were diluted by an additional factor of 2.5 .

Immunofluorescence microscopy. In a preliminary series of experiments we investigated various protocols for fixation. These included fixation in cold methanol $\left(-20^{\circ} \mathrm{C}\right)$, formaldehyde $(4 \%)$, and mixtures of formaldehyde and glutaraldehyde $(0.1-0.4 \%)$. Following aldehyde fixation, cells were extracted in either ethanol or Triton X-100 $(0.25 \%)$. The localization of GAP-43 with each of these protocols was comparable. The following protocol was used for most experiments because of its reproducibility and low background.

Hippocampal cultures were fixed for $20 \mathrm{~min}$ in $4 \%$ formaldehyde in PBS containing $0.12 \mathrm{M}$ sucrose, permabilized in ethanol, and rinsed in PBS. They were blocked with $10 \%$ BSA for $1 \mathrm{hr}$ at $37^{\circ} \mathrm{C}$, then exposed to primary antibody (diluted in $1 \% \mathrm{BSA}$ in PBS) overnight at $4^{\circ} \mathrm{C}$. The following primary antibodies were used in this study: 9-1E12, a mouse monoclonal anti-GAP-43 antibody $(1: 4000)$; an affinity-purified rabbit antiserum directed against GAP-43 (1:500); AP14, a mouse monoclonal antibody directed against MAP2 (1:200); and an affinity-purified rabbit antiserum directed against synapsin 1 (1:25). The cultures were then rinsed with PBS and incubated for $1 \mathrm{hr}$ at $37^{\circ} \mathrm{C}$ with the appropriate secondary antibodies, either goat anti-mouse IgG (1:400, Cappel Laboratories, Cochranville, PA) or goat anti-rabbit IgG (1:200, Cappel Laboratories), labeled either with fluorescein or rhodamine. The cultures were then rinsed with PBS and mounted in PBS:glycerol (1:1) containing $0.05 \% p$-phenylenediamine (Johnson et al., 1981). For double-label experiments, the 2 primary antibodies were incubated together, as were the 2 secondary antibodies. In some experiments rhodamine-labeled wheat germ agglutinin $(40 \mu \mathrm{g} / \mathrm{ml}$, Vector Laboratories) was included with the secondary antibody to localize the Golgi apparatus (Virtanen et al., 1980; Tartakoff and Vassali, 1983).

Cultures were photographed with Tri-X film, which was developed in Diafine. There were frequently large differences in the intensity of the fluorescence associated with different regions of the cells, which were difficult to capture in a single photographic print. In some cases, prints were made so that they illustrated these differences in fluorescence intensity as accurately as possible; in other cases, prints were underexposed to reveal faintly fluorescent structures (as described in the figure legends).

\section{Results}

\section{Development of hippocampal neurons in culture}

As initially described by Dotti ct al. (1988), hippocampal ncurons in culture pass through 4 distinct developmental stages that lead to the acquisition of their mature, polarized form (Fig. 1). Shortly after attachment to the substratum, the neuronal cell bodies become surrounded by flattened lamellipodia (developmental stage 1). The lamellipodia then condense at several discrete points, and from these condensations short, "minor processes" emerge (developmental stage 2). After an interval averaging about $12 \mathrm{hr}$, one of the minor processes begins to grow rapidly, becoming many times longer than the others within just a few hours and acquiring axonal characteristics (developmental stage 3). With the outgrowth of the axon, the cell has become morphologically and functionally polarized. A few days later, the remaining minor processes begin to elongate, but at a slower rate, and acquire the properties of dendrites (developmental stage 4). We examined the distribution of GAP-43 immunoreactivity in neurons at each of these stages.

\section{Characterization of antibodies and reproducibility of staining}

Two antibody preparations were used for identifying GAP-43 on Western blots and for immunocytochemistry of cultured hippocampal neurons. Monoclonal antibody 9-1E12 was raised in mice immunized with GAP- 43 which had been partially purified from neonatal rat brain. This antibody specifically recognizes both soluble and membrane bound GAP-43, in all isoforms resolvable by isoelectric focusing (D. J. Schreyer and J. H. P. Skene, unpublished observations). A rabbit antiserum, prepared against neonatal rat brain GAP-43 purified by preparative isoelectric focusing and preparative SDS gel electrophoresis (Basi et al., 1987), was also used. Both antibodies recognized a single band corresponding to GAP-43 on Western blots of electrophoretic gels of $3 \mathrm{~d}$ postnatal hippocampus and of $8 \mathrm{~d}$ hippocampal cultures (Fig. 2).

Because immunofluorescence is not a quantitative technique, we took great care to confirm each of the principal observations of this study using cells from many independent culture preparations. The photographs selected are representative of the results obtained in every experiment. Any aspects of the GAP. 43 staining that varied are described in the text.

\section{Distribution of GAP-43 and the development of neuronal polarity}

The distribution of GAP-43 at early stages of neuronal development in culture is illustrated in Figure 3. During stage 1, before the initiation of process outgrowth, GAP-43 immunoreactivity was already detectable in the majority of hippocampal neurons. The distribution and intensity of staining varied somewhat from cell to cell, as illustrated by the 3 cells shown in Figure 3, $A-C$. In some cases, GAP-43 immunoreactivity was restricted to a single spot occupying approximately a third of the cytoplasm (Fig. 3A). The location of this staining appeared to be in the region of the Golgi complex (see below). In addition to the GAP-43 immunoreactivity in the cell body, there were 
Figure 1. Stages of development of hippocampal neurons in culture. The approximate times at which cells enter each of the stages are indicated. From Dotti et al. (1988).

\author{
Stage: Lam
}
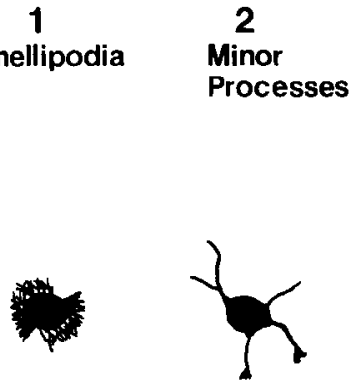

Days in Culture:
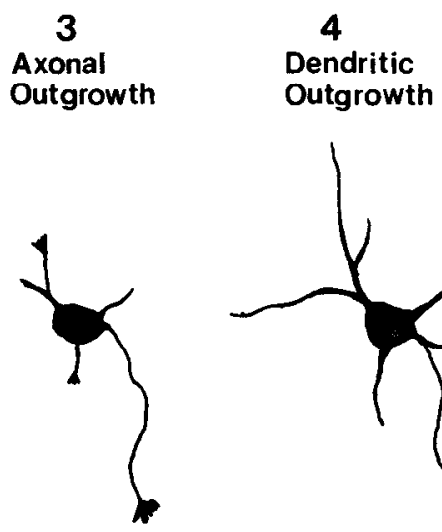

1.5

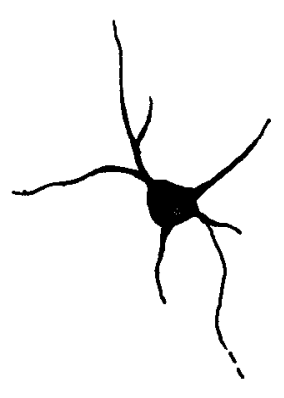

4

often focal concentrations of GAP-43 within the lamellipodia (Fig. 3, B, C). Sometimes, as in Figure $3 B$, this staining closely coincided with condensations visible by phase-contrast $\mathrm{mi}$ croscopy; in other cases (Fig. 3C), a relationship was less obvious.

During the transition between stage 1 and stage 2 , as the lamellipodia condensed at discrete sites along the cell's circumference to form the growth cones of minor processes, there was a concentration of GAP-43 immunoreactivity at these sites. A cell in this transitional stage is illustrated in Figure $3 D$. This neuron had 2 well-defined growth cones and a third which was just beginning to form. At this, the earliest stage of process formation, GAP-43 was already concentrated in each of these newly emerging growth cones. The accumulation of GAP-43 at discrete sites within the lamellipodia may actually precede the formation of minor process growth cones. For example, in the cell illustrated in Figure 3D, a concentration of GAP-43 was observed near the base of the lamellipodia, where there was not yet evidence of process formation. In stage 2 cells the minor processes tend to be spaced at equal intervals along the cell circumference (Dotti et al., 1988). Thus, it is probable that another process would have emerged from this cell near the site of GAP-43 concentration.

Figure 3,E, $F$ illustrates neurons at stage 2 of development, when minor processes are fully formed. At this stage, the level of GAP-43 expression varied considerably, but the immunoreactivity present was predominantly localized to the growth cones of these processes. In most instances, GAP- 43 was about equally concentrated in each of the growth cones, but occasionally 1 or 2 of the minor processes completely lacked GAP-43 immunoreactivity (Fig. $3 F$ ). In addition to its concentration in growth cones, some GAP-43 could be detected within the cell body and as puncta along the processes.

The first morphological evidence of ncuronal polarity arises during the transition between stages 2 and 3 , when one minor process becomes significantly longer than the others (Dotti et al., 1988; Goslin et al., 1989). Cells in this transitional phase are relatively rare because the transition occurs rapidly. Nevertheless, such cells can be identified in fixed cultures, based on the relative length of their processes (Goslin et al., 1989; G. Banker and C. Dotti, unpublished observations). The cell illustrated in Figure $4, A, B$ is representative of cells at the earliest stage when the presumptive axon can be distinguished. In most cases, when this degree of asymmetry has arisen, this process

continues to elongate, becoming the axon. GAP-43 was highly concentrated in the growth cone of the presumptive axon. The cell shown in Figure $4, C, D$ was fixed at a slightly later stage of axonal outgrowth. GAP-43 was strikingly concentrated in its growth cone and there was little detectable immunofluorescence in the growth cones of the remaining minor processes or along the axon. Thus, at the earliest stages in the development of polarity, when the axon could first be identified, GAP-43 was already preferentially distributed to the axon.

Subsequent development during stage 3 is marked by the continued elongation of the axon, with little net growth of the remaining minor processes (Fig. 4, $E, F$ ). As at earlier stages of axonal outgrowth, GAP-43 was highly concentrated in the growth cone of the developing axon, with some particulate staining observed within the shaft of the axon. In some cases, there was also staining of the growth cones of minor processes but to a much lesser extent than of the axonal growth cone. The intense staining of axonal growth cones was not the result of a size difference. Many small axonal growth cones were nevertheless brightly stained, whereas even large growth cones of minor processes were less intensely stained. Moreover, proteins that are present in both types of growth cones, such as actin and the $13 \mathrm{H} 9$ antigen, give staining of roughly equal intensity (Goslin et al., 1989).

Although the most consistent characteristic of cells during stage 3 of development was the high concentration of GAP-43 in the axonal growth cone, some neurons did not exhibit this feature. Since axonal outgrowth by hippocampal neurons is irregular, consisting of periods of active elongation followed by intervals of inactivity (Dotti et al., 1988), it is possible that the axons of such cells were not growing at the time of fixation. The minor processes of stage 3 cells exhibited significant variation in the amount of GAP-43 in their growth cones. In some cells, the minor processes appearcd to bc complctcly devoid of GAP43 immunoreactivity (Fig. 4, C, D), while in others the growth cones of the minor processes were stained to about the same extent as at stage 2 (Fig. $4, E, F$ ).

\section{Distribution of GAP-43 at later stages of development}

Figure 5 shows the distribution of GAP-43 in a single cell early in stage 4 of development, when dendritic elongation begins. This cell was completely isolated, so that the full length of its axon and its entire dendritic tree were readily visible (Fig. $5 A$ ). As is typical of stage 4 cells, GAP-43 had become segregated to 
the axonal domain (Fig. $5 B$ ), but the dendrites and dendritic growth cones had lost GAP-43 immunoreactivity. The axonal identity of the GAP-43-containing processes has been previously established by their lack of MAP2, a dendritic marker (Goslin et al., 1988). In the longer axonal branch (Fig. 5, $C-E$ ), a clear gradient of GAP-43 immunoreactivity was evident; it was highest in the distal axon and growth cone, and declined with increasing proximity to the soma. Within approximately $150 \mu \mathrm{m}$ of the cell body, GAP-43 immunoreactivity was undetectable (Fig. $5 C$ ). In the shorter axonal branch, GAP-43 was present only in the growth cone and the portion of the axon immediately proximal to it. As this example illustrates, the selective segregation of GAP-43 to the axonal domain could be observed even in the absence of cell-cell contact.

By the end of the first week in culture, GAP-43 had become uniformly distributed along the entire axon (Fig. 6, $A, B$ ). As is typical of hippocampal neurons in culture, the axon of the cell shown arose from the base of a short process. GAP- 43 filled the base of this process and entered the axonal branch but was completely excluded from the branch with dendritic properties. In contrast, MAP2 was present in the base of this process and entered the dendritic branch but was completely excluded from the axonal branch (Fig. $6 B$, inset). Figure $6, C, D$ shows a portion of the axonal network from a culture maintained for $5 \mathrm{~d}$. Virtually every axon that could be detected by phase-contrast microscopy appeared to contain GAP-43, which was homogeneously distributed along its length. We observed no apparent decrease in the staining of the axons of hippocampal neurons maintained in culture for up to $30 \mathrm{~d}$ (Fig. 6, E, F).

\section{Changes in the distribution of GAP-43 in axonal growth cones}

The distribution of GAP-43 in distal axons and axonal growth cones was examined in detail in cells maintained from 1 to 30 $\mathrm{d}$ in culture. During the first day or two in culture, labeling within the axon was concentrated almost entirely at the growth cone, with only occasional, lightly stained elements present within the axon proper (Fig. 7, $A-C$ ). At this stage, axonal growth cones were typically large and broad. GAP- 43 was highly concentrated in the central portion of such growth cones (Fig. $7 A, 1 \mathrm{~d}$ in culture). In slightly older cells (Fig. $7, B, C$ ), more staining of the periphery of growth cones was observed, including filopodia and lamellipodia.

With increasing time in culture, axonal growth cones became smaller and narrower, and lost the broad lamellipodial extensions. During this period, the pattern of GAP-43 immunostaining became more homogeneous. GAP-43 immunoreactivity completely filled the growth cone and extended proximally into the axon shaft and filopodia (Fig. 7, $D-G$ ). By $6 \mathrm{~d}$ (Fig. $7 G$ ), homogeneous staining was evident along the entire axon. In regions of the axon that exhibited homogeneous staining, there frequently was a pronounced increase in the intensity of GAP43 immunoreactivity at sites of contact with other axons (Fig. 7, $E, F)$.

In older cultures, as the neuronal network became denser, most axons grew along other fibers, making visualization of growth cones more difficult. The growth cones of the axons present in such fascicles were often small, without obvious filopodia or lamellipodia. Nonetheless, such growth cones could be readily recognized by their high concentration of GAP-43 (Fig. $7 H$ ). By growing cells at low densities, it was sometimes possible to observe free growth cones even after $2-4$ weeks in

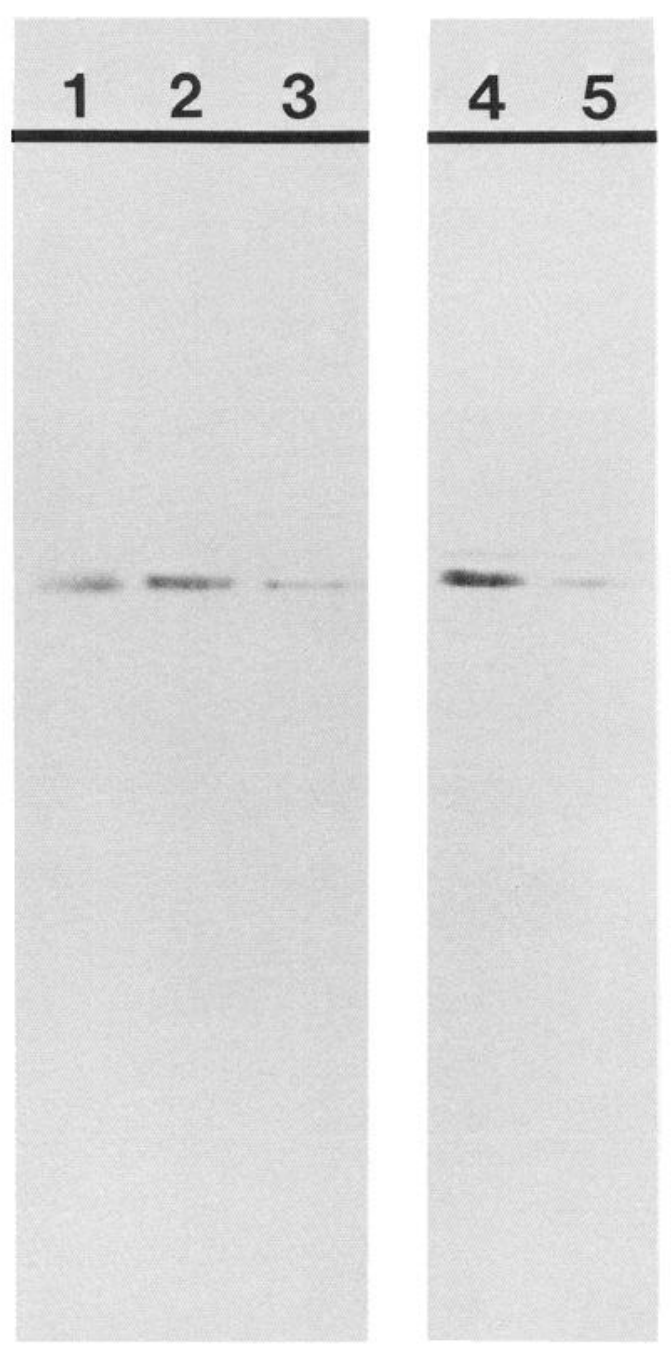

Figure 2. Immunoblots of hippocampal cells probed with monoclonal antibody 9-1E12 (lanes 1-3) or rabbit antiserum against GAP-43 (lanes 4 and 5). Lane 1, partially purified GAP-43; lanes 2 and 4, rat hippocampus, $3 \mathrm{~d}$ postnatal; lanes 3 and 5,8 -d-old hippocampal cultures.

culture (Fig. 7, $I, J$ ). Their morphology and content of GAP-43 were similar to those in cultures examined after 1 week.

\section{Comparison of the distribution of GAP-43 and synapsin 1}

Based on the distribution of immunoreactivity in sections of adult brain, it has been suggested that GAP-43 may be selectively concentrated in presynaptic terminals (Oestreicher and Gispen, 1986). In contrast, in hippocampal neurons GAP-43 appeared to be uniformly distributed along axons in hippocampal cultures, even after several weeks in culture.

To examine this issue in more detail, we used double-label immunofluorescence to localize GAP-43 and the synaptic vesicle antigen synapsin 1 simultaneously (De Camilli et al., 1983) in hippocampal cultures at different times after the onset of synaptogenesis (Fig. 8). Although both antibodies stained axons exclusively, the pattern of staining was quite different. As described above, the GAP-43 immunoreactivity associated with axons was distributed homogeneously along their lengths, while the distribution of synapsin 1 immunoreactivity was punctate. Puncta were restricted to sites of contact between axons and dendrites or cell bodies (Lindsley et al., 1987). 

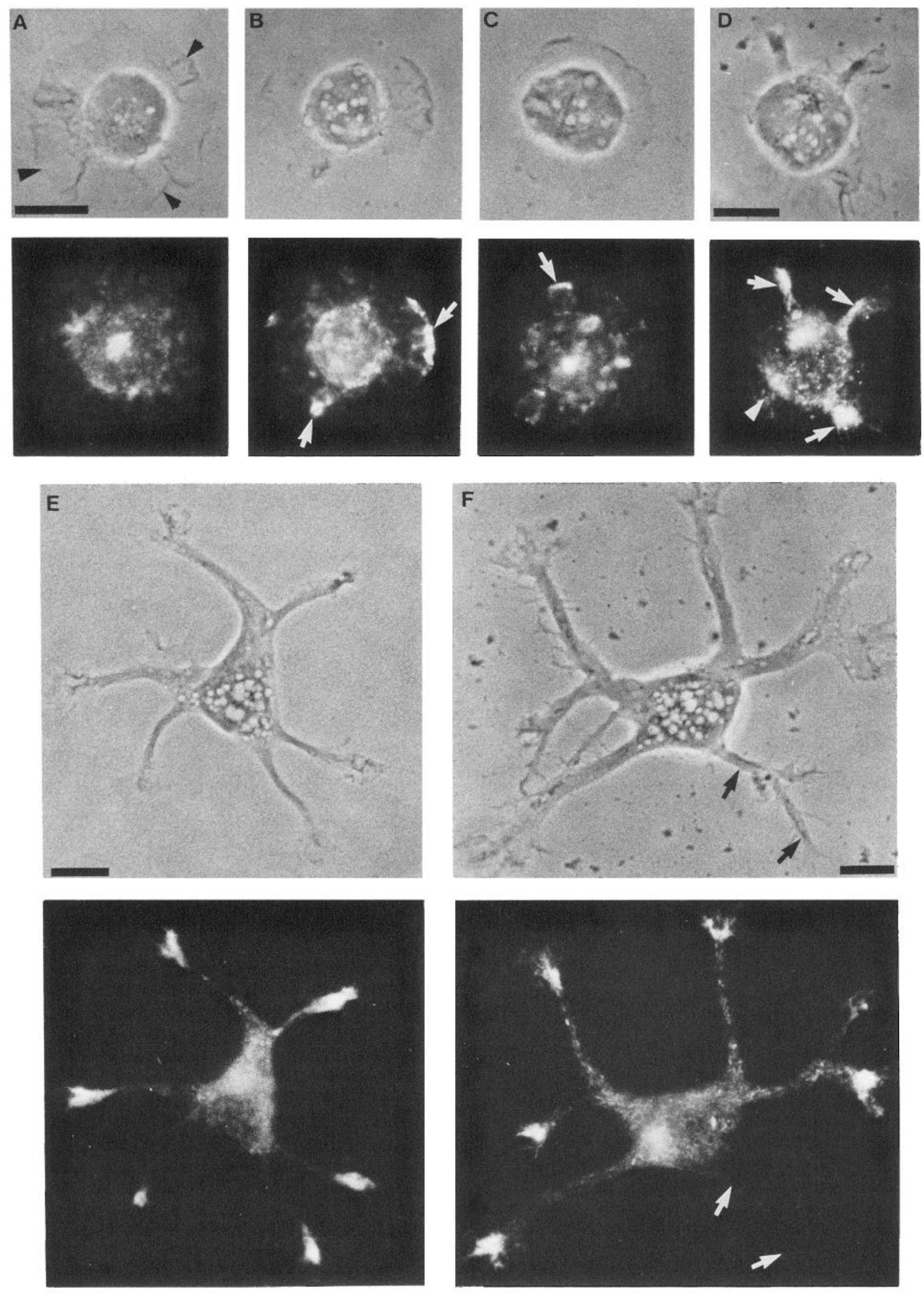

Figure 3. Distribution of GAP-43 at early stages in the development of hippocampal neurons in culture. Cells at stage 1 of development ( $A-C)$ are surrounded by flattened lamellipodia (arrowheads in $A$ ) but lack processes. In some cases, the GAP-43 immunoreactivity in such cells was restricted to a single spot in the cell body $(A)$; in other cases, there were also focal accumulations of GAP-43 in the lamellipodia (arrows in $B$ and 

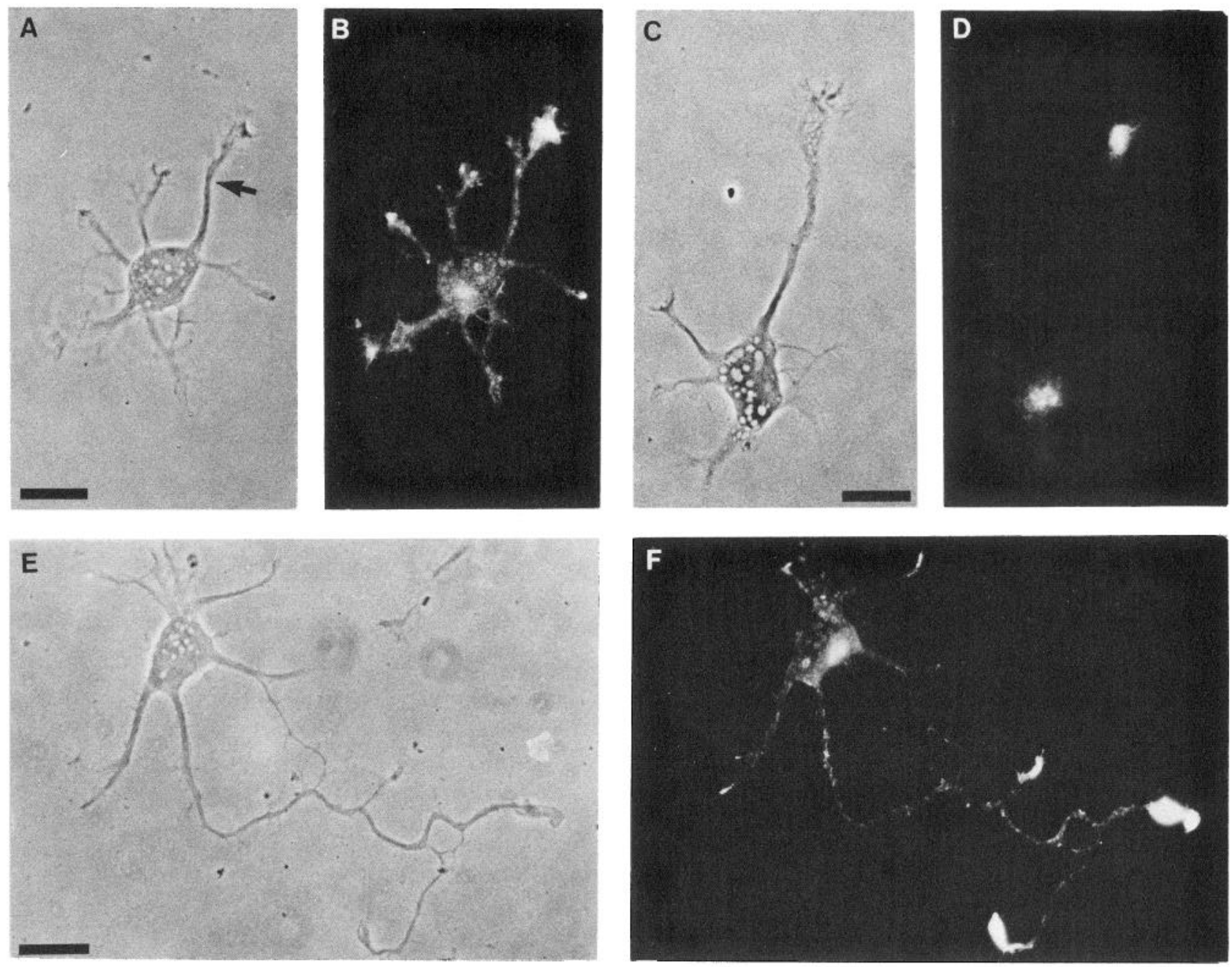

Figure 4. Distribution of GAP-43 during the early stages of axonal outgrowth. The phase-contrast micrographs $(A, C$, and $E)$ illustrate the formation of the axon and its subsequent elongation. The corresponding fluorescence micrographs $(B, D$, and $F)$ show the distribution of GAP-43 at each of these stages. As soon as the axon could be recognized (arrow in $A$ ), GAP-43 was highly concentrated in its growth cone $(B)$. GAP-43 remained concentrated in the axonal growth cone during the further course of axonal elongation $(D, F)$. In many cases, faint particulate staining could also be detected within the axon itself (best seen in $F$ ). The concentration of GAP-43 in the growth cones of the minor processes varied during this period of development. GAP-43 was observed in some, but was undetectable in others. The cells shown ranged in age from 12-36 hr. Scale bar; $15 \mu \mathrm{m}$.

\section{Association of GAP-43 with the Golgi complex}

In the course of these studies, we observed a striking pattern of GAP-43 immunoreactivity in neuronal cell bodies. This labeling, which was present at all stages of development, appeared to be restricted to the region of the cell body containing the Golgi complex. To examine this more carefully, we compared the distribution of GAP-43 with the distribution of intracellular binding sites for wheat germ agglutinin (WGA), a lectin that selectively labels the Golgi region (Virtanen et al., 1980). In hippocampal neurons, WGA gives staining comparable to that obtained with anti-Golgi antibodies (unpublished observations).

During developmental stages 1-3, the Golgi apparatus, visualized by its affinity for WGA, appeared as a single, intensely stained spot eccentrically placed in the cytoplasm (Fig. 9). The location of the Golgi complex had no apparent relationship to the site of origin of the axon. In every neuron, binding sites for WGA colocalized with GAP-43 in the cell body but were absent from the growth cones of axons and of minor processes. No GAP-43 immunoreactivity could be detected in non-neuronal

C). Processes emerge during the transition between stages 1 and $2(D)$; GAP-43 was concentrated in newly forming growth cones as soon as they arose (arrows in $D$ ). An additional accumulation of GAP-43 in this cell (arrowhead) may represent a site from which another process would have emerged. Cells at stage 2 of development $(E, F)$ have 4-6 fully formed "minor processes." GAP-43 was most concentrated in their growth cones, but fainter particulate immunoreactivity could also be detected within the processes. In most instances, GAP-43 was about equally concentrated in the growth cones of each of the minor processes. Occasionally the growth cones of some minor processes lacked GAP-43 immunoreactivity (arrows in F). The content of GAP-43 varied considerably at these stages of development. We have chosen stage 2 cells in which the amount of GAP-43 immunoreactivity was particularly high to better illustrate its distribution, especially its relative concentration in each growth cone. Scale bars, $5 \mu \mathrm{m}$. Bar in $A$ applies to $C$ and $E$ as well. 
A

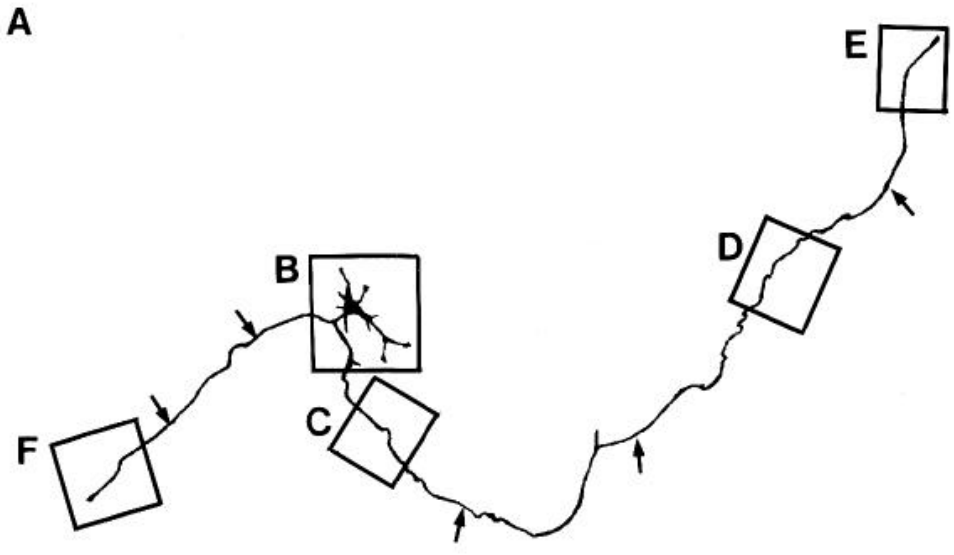

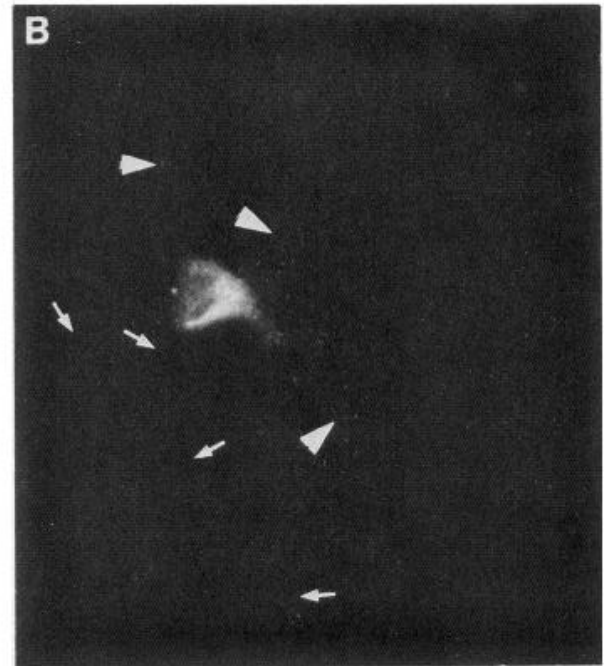
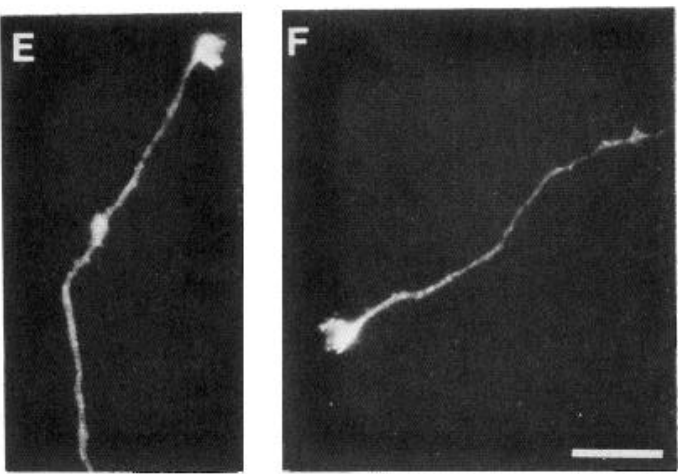

Figure 5. Distribution of GAP-43 in a single, isolated hippocampal neuron after $3 \mathrm{~d}$ in culture. The drawing in $A$ illustrates the cell in its entirety, including the cell body, dendrites, and axon (arrows). The axon bifurcated near its origin, giving rise to a long and a short branch. The distribution of GAP-43 in selected regions of the cell is shown in the fluorescence photomicrographs $(B-F)$, which correspond to the areas indicated in the drawing. As shown in $B$, GAP-43 was present in the cell body but could not be detected in the dendrites (arrowheads) or in the proximal axon (arrows). A gradient of GAP-43 immunoreactivity was observed in the longer axonal branch $(C-E)$. GAP-43 was most concentrated in the axonal growth cone $(E)$, decreasing in intensity in the distal to proximal direction. It could not be detected within $150 \mu \mathrm{m}$ of the soma. In the shorter axonal branch, GAP-43 was present only near the growth cone. Scale bars: $A, 100 \mu \mathrm{m} ; F, 20 \mu \mathrm{m}$.

cells (Fig. 9E), either in the region of the Golgi complex or elsewhere. As expected, WGA labeled the Golgi complex in nonneuronal cells (Fig. 9F). Beginning at about $3 \mathrm{~d}$ in culture, the configuration of the Golgi complex began to change, expanding to form a circle that often fully surrounded the nucleus by day 5. As illustrated in Figure 10, GAP-43 remained associated with the Golgi complex at these later stages of development.

The known properties of GAP-43 suggest that it is a peripheral membrane protein, which would not be expected to undergo processing in the Golgi complex (Basi et al., 1987). Synapsin 1 is also a peripheral membrane protein, and it becomes associated with the cytoplasmic face of vesicles that are selectively transported into the axon (DeCamilli et al., 1983; Nestler and Greengard, 1986; Baitinger and Willard, 1987; T. Fletcher, P. DeCamilli, and G. Banker, unpublished observations). To determine if synapsin 1 also is concentrated in the region of the Golgi complex prior to its transport into the axon, we compared the distribution of these 2 proteins within neuronal somata (Fig. 11). Throughout development, GAP- 43 was highly concentrated in the Golgi region, while the cell bodies were virtually devoid of synapsin 1 immunoreactivity.

The region of the cell body containing the Golgi complex is also an area where endocytotic vesicles accumulate. When such vesicles were labeled by brief exposure of living cells to WGA, they colocalized with the GAP-43 staining in the cell bodies of stage 3 neurons (K. Goslin, unpublished observations). In older cells, however, they were distributed irregularly throughout the cell body (data not shown). This did not resemble the pattern of labeling with anti-GAP-43.

\section{Discussion}

We have previously shown that in cultured hippocampal neurons with well-differentiated axons and dendrites, GAP-43 is selectively distributed to the axonal domain (Goslin et al., 1988). In the present study, we investigated how this selective distribution arises during neuronal development. Initially, in unpolarized cells, GAP-43 is equally distributed among the growth cones of the minor processes. Then, as one of the minor processes begins to grow rapidly and acquires axonal characteristics, GAP-43 becomes preferentially concentrated in its growth cone. Subsequently, GAP-43 is lost from the growth cones of the remaining minor processes, as they acquire dendritic properties. These observations provide new insights regarding the distribution of an axonally targeted membrane protein during key stages in the development of neuronal polarity and raise the possibility that GAP-43 may itself play a role in these events. 

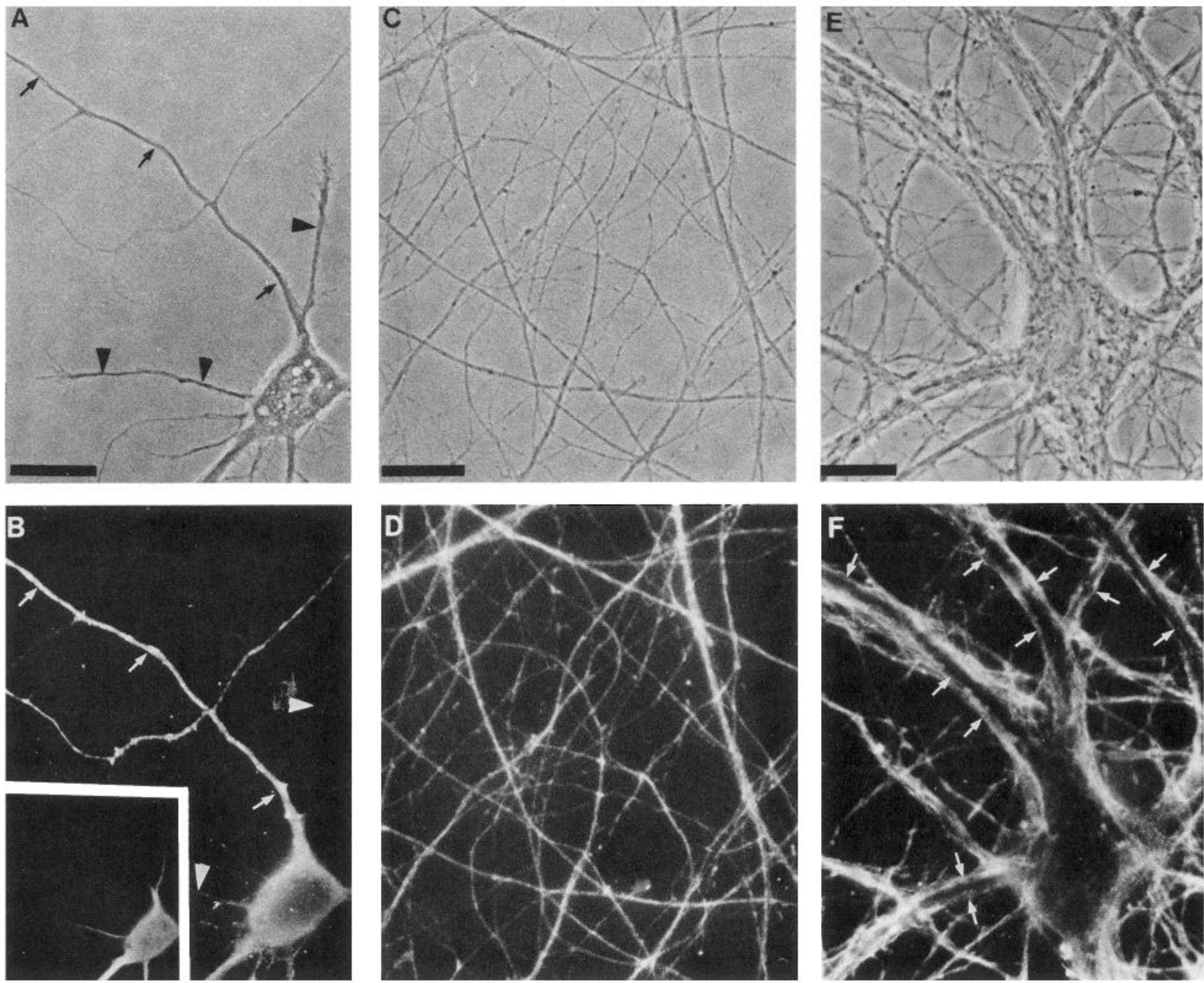

Figure 6. Phase-contrast $(A, C$, and $E)$ and fluorescence micrographs $(B, D$, and $F)$ illustrating the distribution of GAP-43 during later stages of neuronal development. $A$ and $B$ illustrate a neuron after 6 days in culture. At this stage, GAP-43 was uniformly distributed throughout the axon (arrows), extending proximally to its origin from the cell body. The dendrites and their growth cones lacked detectable immunoreactivity (arrowheads). The axon of this cell arose from the base of a short process. GAP-43 filled the base of this process and entered the axonal branch but was excluded from the branch with dendritic properties. MAP2 was present in the base of this process and entered the dendritic branch but was excluded from the axonal branch (inset in $B$ ). At this early stage of dendritic development, the MAP2 staining did not extend the entire length of the dendrites. A representative example of an axonal field from a $5 \mathrm{~d}$ culture is shown in $C$ and $D$. Virtually every axon was homogeneously stained. This distribution of GAP-43 was maintained during the further course of neuronal maturation in culture as illustrated by the 28 -d-old cell in $E$ and $F$. GAP-43 was homogeneously distributed throughout the axons but was absent from the dendrites. The dendrites were frequently outlined by brightly stained axons running along their surfaces (arrows) but were themselves devoid of GAP-43 immunoreactivity. Scale bars: $A$ and $C, 20 \mu$ m; $E$, $15 \mu \mathrm{m}$.

\section{Segregation of GAP-43 to the axonal domain}

The segregation of GAP-43 to the axonal domain begins during the transition between stages 2 and 3 . The approximately equal distribution of GAP-43 among the minor processes prior to this transition is consistent with the idea that the eventual identity of the minor processes has not yet been determined at stage 2; no one process has been specified as the axon (Dotti and Banker, 1987; Dotti et al., 1988; Goslin and Banker, 1989).

It is during this transition between stage 2 and 3 that the first morphological indication of polarity arises, when one minor process becomes obviously longer than the others. Analysis of the responses of stage 3 neurons to transection of the axon indicates that once the length of one process exceeds that of all others by $10 \mu \mathrm{m}$ or more, it is specified as the axon and rapidly acquires axonal properties (Goslin and Banker, 1989). At this stage of development axons grow at rates of $10-20 \mu \mathrm{m} / \mathrm{hr}$ (Dotti et al., 1988), suggesting that the acquisition of polarity during normal development could occur in less than an hour. This has been confirmed in preliminary observations of normal cells undergoing the transition between stages 2 and 3 (Banker and Dotti, unpublished observations). Because GAP-43 is already selectively concentrated in the axonal growth cone when polarity can first be detected morphologically, its preferential accumulation presumably occurs in a similarly short interval.

In general, the loss of GAP-43 from the remaining minor processes occurs later in development, and with a slower time course, than the concentration of GAP-43 in the axonal growth cone. The loss is not complete until about the third day in culture, roughly during the transition between stages 3 and 4 . 

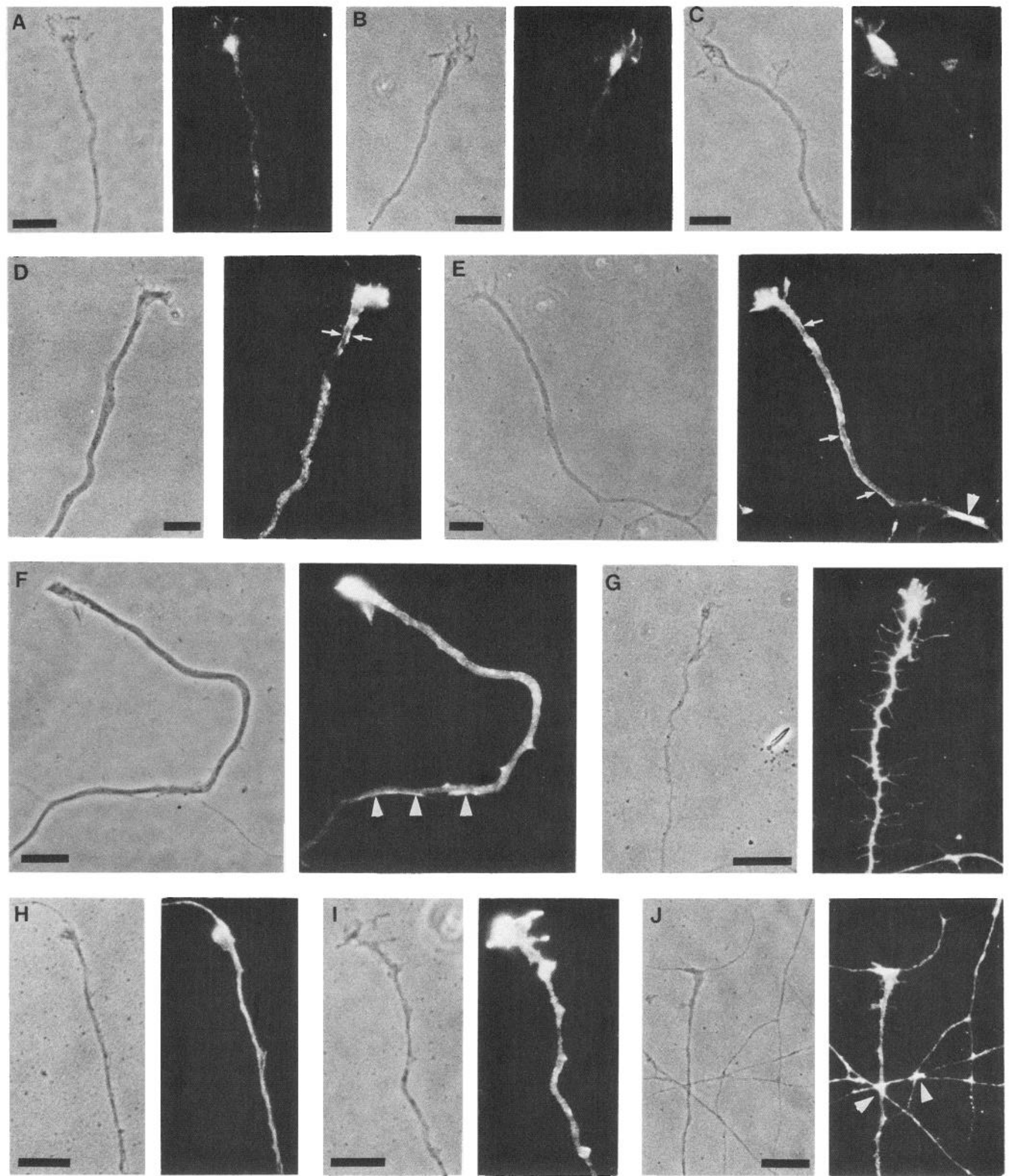

Figure 7. Changes in the distribution of GAP-43 in the axonal growth cone and distal axon during development in culture. Phase-contrast and immunofluorescence views of growth cones ranging in age from 1-30 d. At early stages of development $(A-C)$, axonal growth cones were characterized by a central region, from which a fan-shaped lamellipodium extended. GAP-43 was highly concentrated in this region of the growth cones, but little GAP-43 extended into the lamellipodia. Frequently, as in $B$ and $C$, the distal axon was nearly devoid of immunoreactivity. Occasionally, as in $A$, fine particulate staining within the distal axon could also be observed. Between 2 and $4 \mathrm{~d}$ in culture, there was a change in both the morphology of the growth cones and in the distribution of GAP-43 $(D, E)$. The growth cones became smaller and the lamellipodia less prominent. GAP-43 filled the entire growth cone, including filopodia. In addition, GAP-43 became more concentrated in the distal axon and frequently appeared to be associated with the surface membrane. At some sites, there was a pronounced concentration of GAP-43 along the edges of the axon (arrows in $D$ and $E$ ); in others, homogeneous staining extended across the entire width of the axon. From this stage onward, there was also a marked increase 

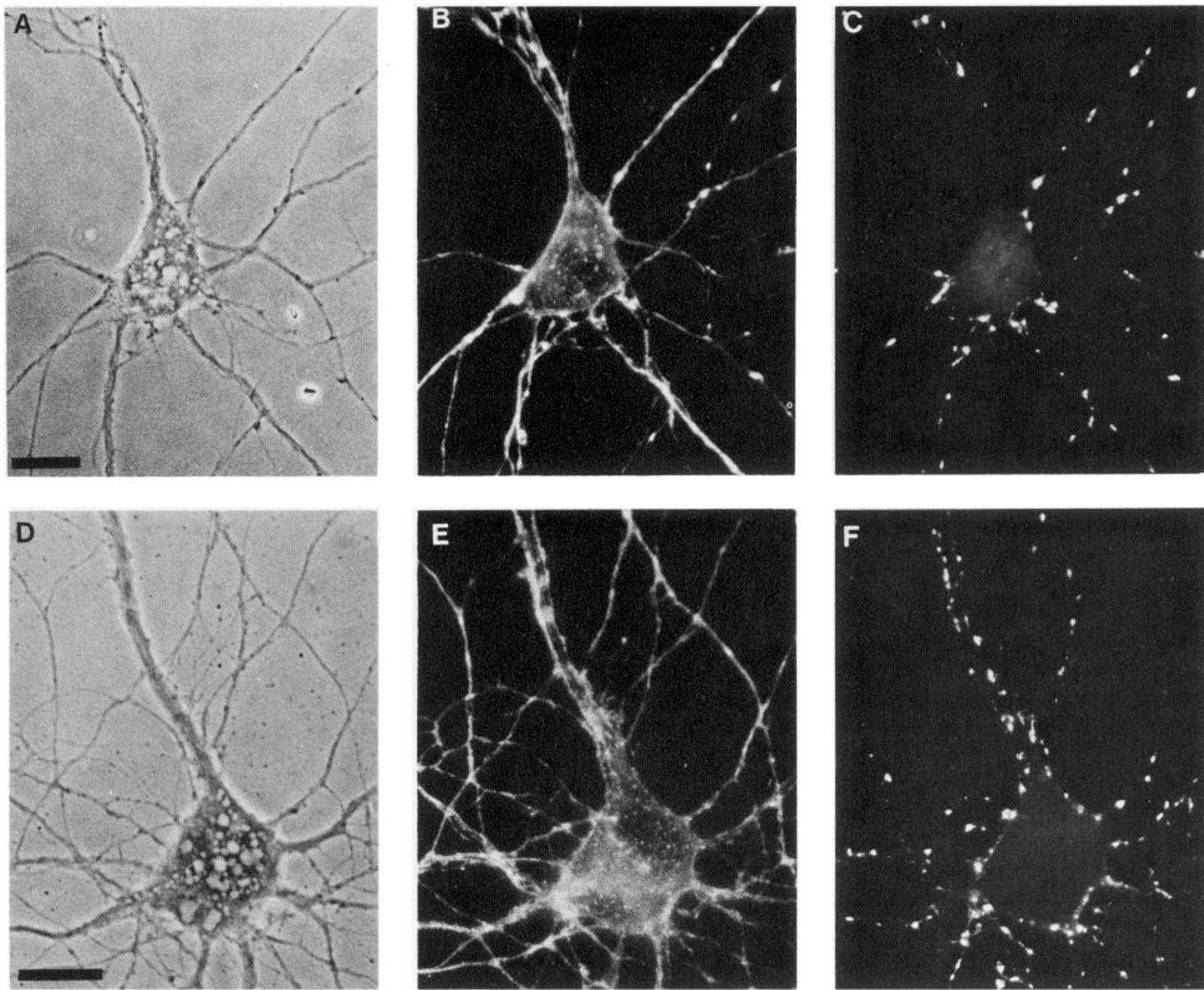

Figure 8. Phase-contrast $(A$ and $D)$ and fluorescence micrographs comparing the distribution of GAP-43 $(B$ and $E)$ and synapsin $1(C$ and $F)$ in 14-d-old hippocampal cultures. Where axons ran along dendrites and cell bodies, GAP-43 was uniformly distributed, whereas synapsin 1 was concentrated in discrete puncta corresponding to synaptic boutons. GAP-43 was also homogeneously distributed in regions of the axonal network where there were no synapses. Such regions contained little or no synapsin 1 immunoreactivity. Scale bars, $15 \mu \mathrm{m}$.

\section{Changes in the distribution of GAP-43 in axons and axonal growth cones}

The further development of hippocampal neurons in culture is marked by 2 significant changes in the distribution of GAP-43 within the axonal domain. First, GAP-43 is initially highly concentrated only in the growth cones at the tips of axons, but subsequently becomes distributed along the entire axon. This progresses in the distal-to-proximal direction, suggesting that GAP-43 may be added to the elongating axon preferentially at the growth cone (see also Meiri et al., 1988). This is consistent with evidence that incorporation of new membrane occurs pre- dominantly at that site (Bray, 1970; Griffin et al., 1981; Pfenninger and Maylie-Pfenninger, 1981).

In observations of cultured sympathetic neurons, Meiri et al. (1988) did not observe a distinct stage during axonal outgrowth when GAP-43 was restricted to the axonal growth cone. Significant levels of GAP-43 are present along the axons of sympathetic neurons throughout development. Although this could result from intrinsic differences between cell types, it might simply reflect differences in the relative maturity of the cells when introduced into culture. More mature neurons, like the sympathetic neurons used by Meiri et al. (1988), may already have passed through the stage when GAP-43 is largely restricted

in GAP-43 immunoreactivity at sites of contact between axons (arrowheads in $E, F$, and $J$ ). By the end of the first week in culture, axonal growth cones had become smaller and somewhat club-shaped $(F, G)$. Staining of the distal axon appeared more homogeneous and intense, and the demarcation between growth cone and distal axon less pronounced. Filopodia along the axon were also stained $(G)$. These characteristics were observed in growth cones up to $30 \mathrm{~d}$ in culture $(H, J)$, including those growing along other axons $(H)$. In preparing several of the photographic prints in this figure, the growth cone region was "burned in" to bring out staining along the axon. $A, 1 \mathrm{~d}$ in culture; $B$ and $C, 1.5 \mathrm{~d} ; D$ and $E$, $2 \mathrm{~d}$; $F, 4 \mathrm{~d} ; G, 6 \mathrm{~d} ; H, 7 \mathrm{~d} ; I, 14 \mathrm{~d} ; J, 30$ d. Scale bars, $10 \mu \mathrm{m}$. 

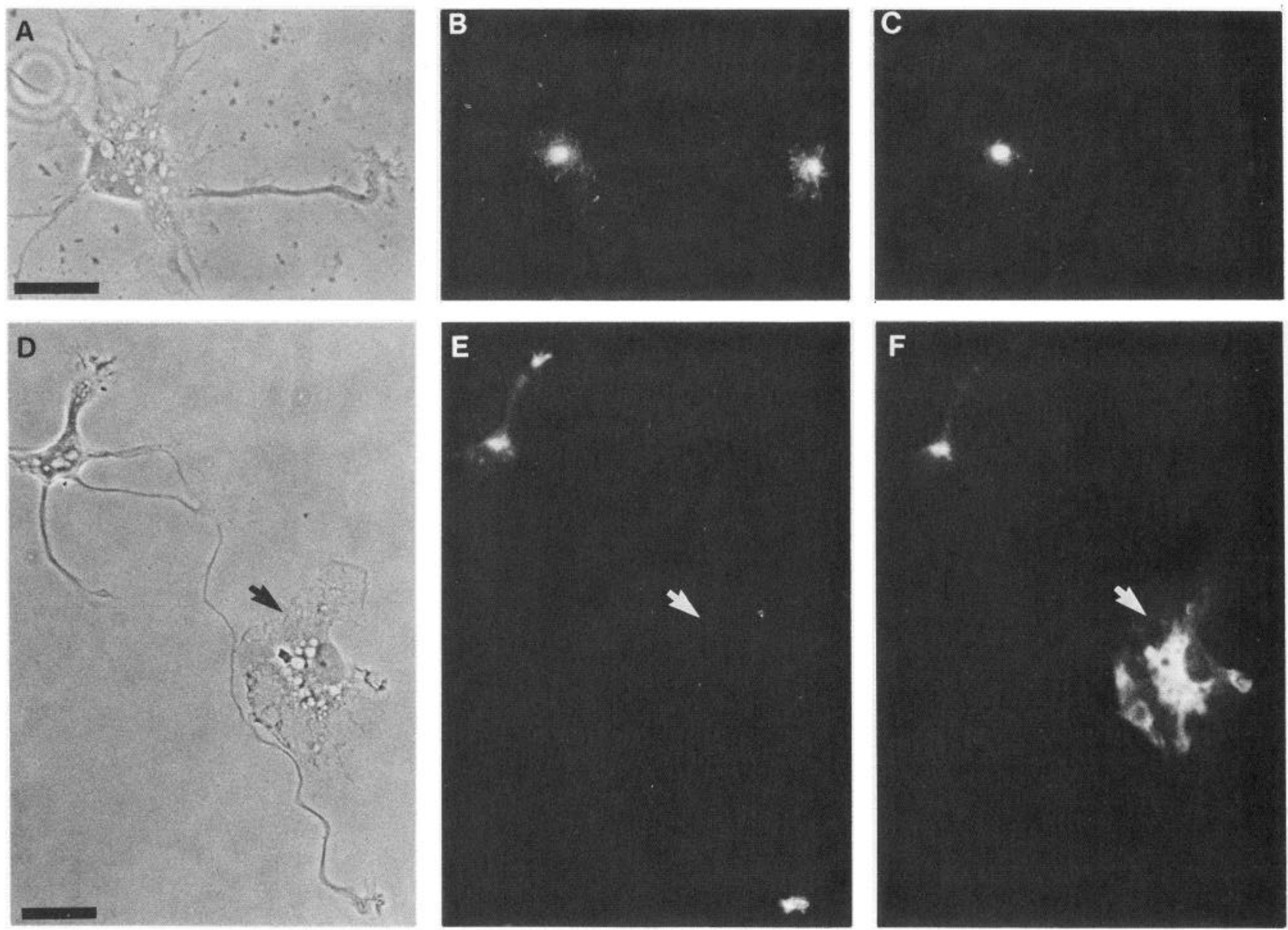

Figure 9. Association of GAP-43 with the Golgi apparatus. Phase-contrast $(A$ and $D)$ and immunofluorescence micrographs illustrating the distribution of GAP-43 ( $B$ and $E$ ) and of intracellular binding sites for wheat germ agglutinin (WGA) $(C$ and $F$ ) during stage 3 of development. The highest concentration of GAP-43 was found in the axonal growth cone and in a single spot within the cell body. GAP-43 could also be detected in the growth cones of some of the minor processes. The concentration of GAP-43 in the cell body colocalized with the Golgi complex, as seen following staining with WGA. Non-neuronal cells lacked GAP-43 immunoreactivity but were stained by WGA (arrows in $D-F)$. $A-C$, 16 hr in culture; $D-F, 1.5 \mathrm{~d}$ in culture. Scale bars, $20 \mu \mathrm{m}$.
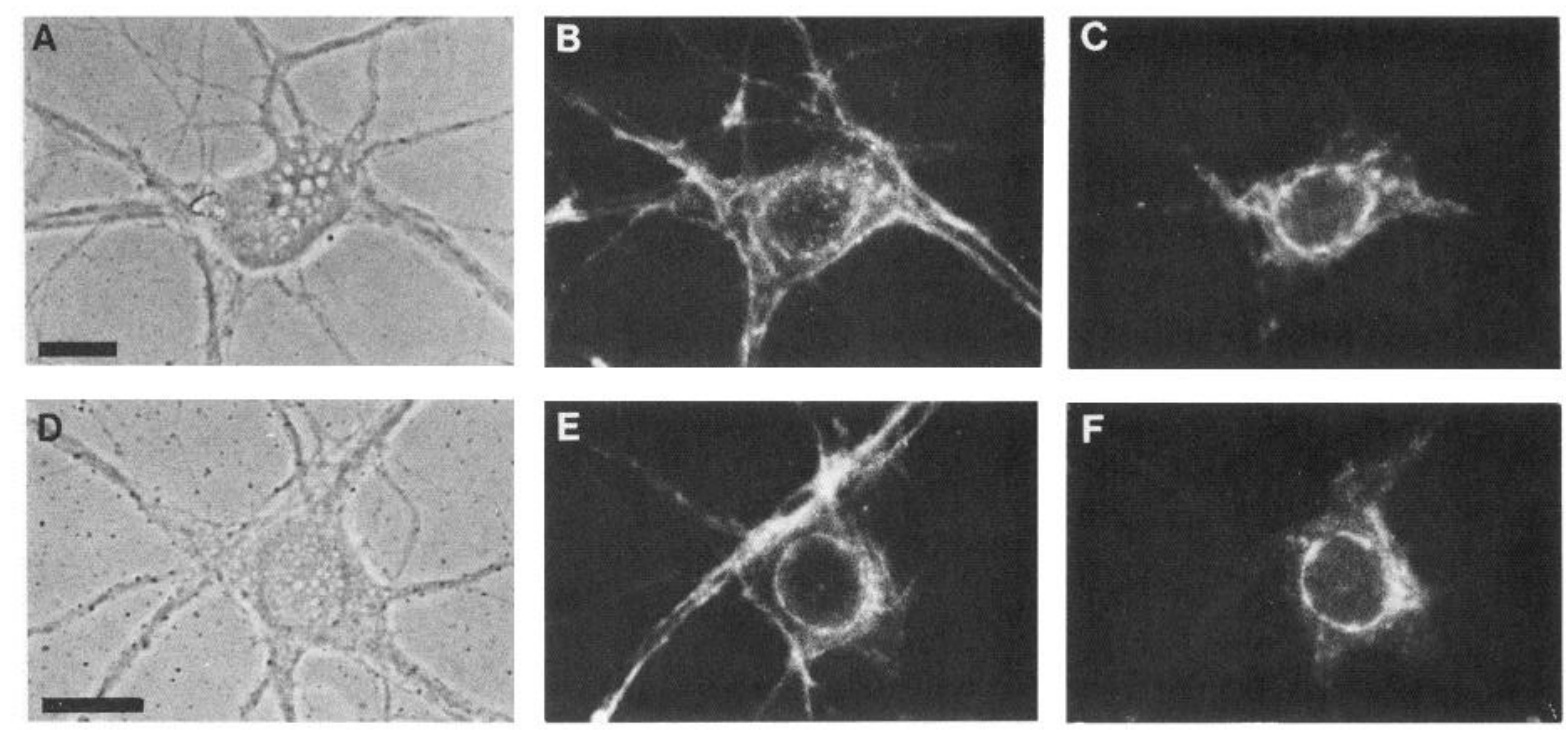

Figure 10. Association of GAP-43 with the Golgi apparatus at later stages of development. Phase-contrast $(A$ and $D)$ and fluorescence micrographs illustrating the distribution of GAP-43 $(B$ and $E$ ) and of intracellular binding sites for WGA ( $C$ and $F$ ). The Golgi apparatus had assumed a perinuclear configuration, as demonstrated by WGA staining. GAP-43 continued to be localized to the Golgi region. The fluorescence micrographs were taken at the focal plane of the Golgi complex; stained axons running along the substrate were, therefore, barely visible. $A-C$, $16 \mathrm{~d}$ in culture; $D-F, 24 \mathrm{~d}$ in culture. Scale bars, $15 \mu \mathrm{m}$. 

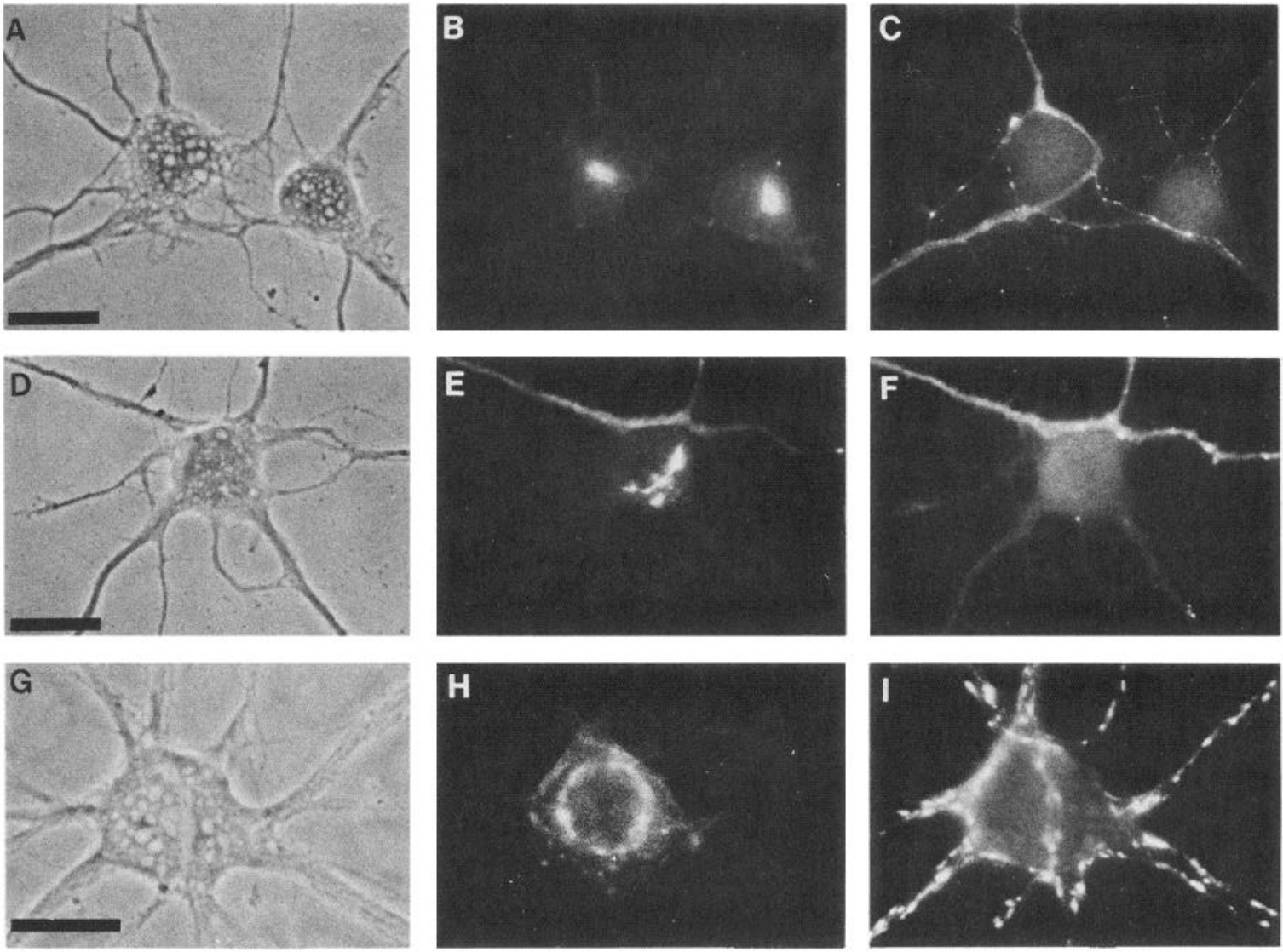

Figure 11. Comparison of the distribution of GAP-43 and synapsin 1 in neuronal cell bodies. Phase-contrast $(A, D$, and $G)$ and fluorescence micrographs illustrating the distribution of GAP-43 $(B, E$, and $H)$ and of synapsin $1(C, F$, and $I)$ after $3 \mathrm{~d}(A-F)$ and $24 \mathrm{~d}(G-I)$ in culture. At all stages of development, GAP-43 stained the Golgi apparatus. The configuration of the Golgi complex changed with age in culture from a single spot $(B)$, to a semicircle partially surrounding the nucleus $(E)$, to a ring encircling the entire nucleus $(H)$. There was no detectable synapsin 1 immunoreactivity associated with the Golgi complex at any stage. The fluorescence micrographs were taken at the focal plane of the Golgi complex, so that axonal staining was not always visible. Scale bars, $15 \mu \mathrm{m}$.

to growth cones. Moreover, since these cells have extended axons in situ at the time they are taken for culture, they probably already express GAP-43 at high levels, which might tend to obscure a selective concentration of GAP-43 in the axonal growth cone.

There were no further changes in the distribution of GAP-43 in axons as they matured, even in cultures maintained for 30 d. This result contrasts with observations in situ, which show that during maturation GAP-43 disappears from axons within fiber tracts but persists in some regions enriched in axon terminals and synapses (Gispen et al., 1985; Oestreicher and Gispen, 1986). It may be that axons in hippocampal cultures more closely resemble terminal axons than axons in fiber tracts. The immediate cellular environment in culture certainly resembles that of a terminal field in situ and the extensive branching of axons in culture is also characteristic of terminal axons. Alternatively, a further maturation of axons may occur in situ that is not attained under our culture conditions.

Oestreicher and Gispen (1986) noted that the pattern of GAP43 immunoreactivity in regions of neuropil was punctate and likened this to the pattern of staining seen by others using antibodies directed against synaptic vesicle proteins (see also Oestreicher et al., 1981; Gispen et al., 1985). In hippocampal cultures the synaptic vesicle antigen synapsin 1 was selectively localized to synaptic boutons and virtually absent from segments between boutons, while GAP-43 was uniformly distributed along the entire axon. Thus, while GAP- 43 may be restricted to neuropil regions containing terminal axons, as Oestreicher and Gispen describe, it need not be exclusively localized to presynaptic boutons, where synaptic vesicles are concentrated.

In addition to changes in the distribution of GAP-43 along the axon, the nature of the axonal staining also changed during development. The staining was initially punctate in appearance, as if GAP-43 were associated primarily with small organelles within axons. As development progressed, the GAP-43 labeling became more homogeneous and extended into filopodia and lamellipodia, which contain little or no intracellular membrane (Yamada et al., 1971; Bunge, 1973). This diffuse pattern might suggest that a portion of the GAP-43 becomes evenly associated with the axonal plasma membrane. In cultured sympathetic neurons (Meiri et al., 1988) and PC12 cells (Van Hooff et al., 1989), GAP-43 immunoreactivity appears to be associated both with intracellular membranous organelles and with the surface membrane. If GAP-43 becomes associated with the surface membrane, this presumably must occur at the cytoplasmic, rather than the extracellular face. GAP-43 lacks potential membrane-spanning domains and sites for $\mathrm{N}$-linked glycosylation 
(Basi et al., 1987) and can be detected immunocytochemically only after permeabilization of the membrane (Meiri et al., 1988; Van Hooff et al., 1989).

The staining in axonal growth cones at early stages in the development of hippocampal neurons is largely confined to their central regions. This portion of the growth cone contains a high concentration of membranous elements resembling smooth endoplasmic reticulum (J. Deitch and G. Banker, unpublished observations; see also Yamada et al., 1971; Bunge, 1973) and is also thought to be the preferential site of membrane addition to the cell surface during axonal elongation (Bray, 1970; Griffin et al., 1981; Pfenninger and Maylie-Pfenninger, 1981). The concentration of GAP-43 within the central region of the axonal growth cones at early stages of development is consistent with its association either with intracellular membranes or with the plasmalemma.

At the time when the pattern of immunostaining suggests that GAP-43 has become associated with the surface membrane, marked concentrations of GAP-43 become evident at sites of contact between axons. Frequently, ligands that cause aggregation of transmembrane proteins by binding to the cell surface concomitantly cause capping of associated proteins, such as fodrin, within the cell cortex (Levine and Willard, 1983). This raises the possibility that the distribution of GAP-43, associated with the cytoplasmic aspect of the surface membrane, may be altered in response to contacts impinging on the extracellular surface.

Cellular mechanisms underlying developmental changes in the distribution of $G A P-43$

We have described a series of changes in the distribution of GAP-43 in developing hippocampal neurons that correlate with key events in their acquisition of polarity. Several distinct cellular mechanisms could contribute to these changes in distribution.

Studies in situ have shown that GAP-43 is initially synthesized as a soluble protein which become associated with neuronal membranes in less than 20 min (Skene and Virag, 1989). This relatively rapid association of GAP-43 with membranes suggests that its attachment to membranes occurs near its site of synthesis in neuronal cell bodies. GAP-43 is transported from the cell body along the axon in the rapid phase of axonal transport (Skene and Willard, 1981a, b). If the intracellular movement of GAP-43 occurs in a vesicle-associated form, as studies of its axonal transport suggest, its selective distribution might arise by a mechanism analogous to that described for the sorting of membrane proteins in polarized epithelial cells (Simons and Fuller, 1985; Griffiths and Simons, 1986). The selective accumulation of GAP-43 in the axonal growth cone which occurs rapidly during the transition between stage 2 and stage 3 would imply that the machinery for selective sorting and transport must also become fully functional at this time.

Recent studies, which provide evidence that the association of GAP-43 with neuronal mcmbranes is mediated by fatty acylation, suggest other possible mechanisms for the changes in the distribution of GAP-43 during development (Skene and Virag, 1989). In isolated growth cones there is a turnover of the acyl moiety of GAP-43, indicating that there may be a dynamic interchange of GAP-43 between a soluble and a membraneassociated form. GAP-43 released from membranes by deacylation might be redistributed within the cell either by diffusion or reacylation and attachment to a mobile population of membrane vesicles. Reacylation of GAP-43 might also provide a mecha- nism for "trapping" the protein in regions with high local concentrations of free fatty acid or locally activated protein : acyl transferases.

The polarity orientation of microtubules within neuronal processes may also contribute to the distribution of GAP-43 during development. During both stages 2 and 3, microtubules in all neurites are oriented uniformly, with their $[+]$-ends directed toward the growth cones (Baas et al., 1989). At about the time of the transition between stages 3 and 4, a population of microtubules arises whose [+]-ends are directed toward the cell body (Baas et al., 1988, 1989). The polarity of microtubules in the axon remains uniform. If GAP -43 had a preferential affinity for an anterograde translocator, such as kinesin, the change in microtubule polarity during the transition between stages 3 and 4 could contribute to the loss of GAP-43 from dendritic processes. The time course of this loss is also compatible with the metabolic turnover time of GAP-43, which has been estimated at $1 \mathrm{~d}$ (Skene and Willard, 1981c).

\section{Concentration of GAP-43 in the region of the Golgi apparatus}

By comparing the pattern of GAP-43 immunoreactivity with that of intracellular binding sites for WGA, we determined that somatic GAP-43 is concentrated in the region of the Golgi complex. Meiri et al. (1988) also noted a perinuclear concentration of GAP-43 in cultured sympathetic neurons that may correspond to staining of the Golgi region.

If confirmed by electron microscopic immunocytochemistry, the association of GAP-43 with the Golgi complex is of considerable interest. GAP-43 is synthesized as a soluble protein and is not known to be glycosylated, but its concentration in the Golgi region might reflect its association with membrane vesicles there prior to sorting and transport into the axon. It should be noted, however, that synapsin 1, which is associated with the cytoplasmic surface of vesicles that are selectively transported into the axon, is not concentrated in the Golgi region.

It is possible, therefore, that the staining observed in the Golgi region is not merely incidental to the intracellular processing of newly synthesized GAP-43 but reflects one functional location of this protein. It is striking that the 2 sites where GAP-43 appears to be most concentrated, the Golgi complex and the axonal growth cone, are both regions of highly dynamic membrane movements, including vesicle fusion and vesicle formation (e.g., Bunge, 1977). In non-neuronal cells, proteins involved in the vectorial transport of membrane vesicles show a similar concentration in the Golgi region and at sites of vesicle addition to the plasma membrane. In view of this, it may be useful to consider a possible involvement of GAP-43 in intracellular membrane traffic (see also Gordon-Weeks, 1989).

\section{Possible function of GAP-43 in the development of polarity}

Because GAP-43 is present in growing axons and axonal growth cones, but is absent from dendrites and dendritic growth cones, we have argucd that it may give axons certain of their unique growth characteristics (Goslin et al., 1988). The properties of GAP-43, including its proposed interactions with intracellular messenger systems (for review, see Benowitz and Routtenberg, 1987; Skene, 1989), suggest that it may play a role in regulating axonal growth. Within the time resolution of the present study, we have shown that the selective accumulation of GAP-43 in one of the minor processes occurs coincidently with the acquisition by that process of axonal growth properties.

Based on the response of cultured hippocampal neurons to 
axonal transection, we have proposed that the specification of neuronal polarity could involve the selective accumulation of a protein that regulates the rate of neurite outgrowth in the growth cone of a single process (Goslin and Banker, 1989). If such a protein were equally concentrated in the growth cones of minor processes during stage 2 , before polarity has been expressed, but became preferentially associated with the axonal growth cone as the axon became significantly longer than the other processes, this could explain why only a single process grows at the rapid rate characteristic of axons. Though there may be many other proteins besides GAP-43 that could influence the rate of growth of the emerging axon, the developmental distribution of GAP43 is just that predicted from our previous results. It is the only protein yet identified that is selectively concentrated in the axonal growth cone and its preferential accumulation in the emerging axon is the earliest known molecular event associated with the development of neuronal polarity.

\section{References}

Baas, P. W., J. S. Deitch, M. M. Black, and G. A. Banker (1988) Polarity orientation of microtubules in hippocampal neurons: Uniformity in the axon and nonuniformity in the dendrite. Proc. Natl. Acad. Sci. USA 85: 8335-8339.

Baas, P., G. Banker, and M. Black (1989) The establishment of microtubule patterns in cultured hippocampal neurons. J. Cell. Biol (in press).

Baitinger, C., and M. Willard (1987) Axonal transport of synapsin 1 -like proteins in rabbit retinal ganglion cells. J. Neurosci. 7: 37233735.

Banker, G. (1980) Trophic interactions between astroglial cells and hippocampal neurons in culture. Science 209: 809-810.

Banker, G., and W. M. Cowan (1977) Rat hippocampal neurons in dispersed cell culture. Brain Res. 126: 397-425.

Bartlett, W. P., and G. A. Banker (1984a) Electron microscopic studics of axonal and dendritic development by hippocampal neurons in culture. I. Cells which develop without intercellular contacts. J. Neurosci. 4: 1944-1953.

Bartlett, W. P., and G. A. Banker (1984b) Electron microscopic studies of axonal and dendritic development by hippocampal neurons in culture. II. Synaptic relationships. J. Neurosci. 4: 1954-1965.

Basi, G. S., R. D. Jacobson, I. Virag, J. Schilling, and J. H. P. Skene (1987) Primary structure and transcriptional regulation of GAP-43, a protein associated with nerve growth. Cell 49: 785-791.

Benowitz, L. I., and E. R. Lewis (1983) Increased transport of 44,000to 49,000 -dalton acidic proteins during regeneration of the goldfish optic nerve: A two-dimensional gel analysis. J. Neurosci. 3: 21532163.

Benowitz, L. I., and A. Routtenberg (1987) A membrane phosphoprotein associated with neural development, axonal regeneration, phospholipid metabolism, and synaptic plasticity. Trends Neurosci. 10: 527-531.

Booher, J., and M. Sensenbrenner (1972) Growth and cultivation of dissociated neurons and glial cells from embryonic chick, rat, and human brain in flask cultures. Neurobiology 2:97-105.

Bottenstein, J. E., and G. H. Sato (1979) Growth of a rat neuroblastoma cell line in serum-free supplemented medium. Proc. Natl. Acad. Sci. USA 76: 514-519.

Bray, D. (1970) Surface movements during the growth of single explanted neurons. Proc. Natl. Acad. Sci. USA 65: 4516-4519.

Bunge, M. B. (1973) Fine structure of nerve fibers and growth cones of isolated sympathetic neurons in cultures. J. Cell. Biol. 56: 713735.

Bunge, M. B. (1977) Initial endocytosis of peroxidase or ferritin by growth cones of cultured nerve cells. J. Neurocytol. 6: 407-439.

Caceres, A., G. Banker, and L. Binder (1986) Immunocytochemical localization of tubulin and microtubule-associated protein 2 during the development of hippocampal neurons in culture. J. Neurosci. 6 : 714-749.

DeCamilli, P., R. Cameron, and P. Greengard (1983) Synapsin 1 (Protein 1), a nerve terminal-specific phosphoprotein. I. Its general distribution in synapses of the central and peripheral nervous system in frozen and plastic sections. J. Cell Biol. 96:1337-1354.

Dotti, C. G., and G. A. Banker (1987) Fxperimentally induced alteration in the polarity of developing neurons. Nature 330: 254-256.

Dotti, C. G., C. A. Sullivan, and G. A. Banker (1988) The establishment of polarity by hippocampal neurons in culture. J. Neurosci. 8: 1454-1468.

Gispen, W. H., J. L. M. Leunissen, A. B. Oestreicher, A. J. Verkleij, and H. Zwiers (1985) Presynaptic localization of B-50 phosphoprotein: The (ACTH)-sensitive protein kinase substrate involved in rat brain polyphosphoinositide metabolism. Brain Res. 328: 381-385

Gordon-Weeks, P. R. (1989) GAP-43-What does it do in the growth cone? Trends Neurosci. 12: 238-240.

Goslin, K., and G. A. Banker (1989) Experimental observations on the development of polarity by hippocampal neurons in culture. $\mathrm{J}$. Cell Biol. 108: 1507-1516.

Goslin, K., D. I. Schreyer, J. H. P. Skene, and G. A. Banker (1988) Development of neuronal polarity: GAP-43 distinguishes axonal from dendritic growth cones. Nature 336: 672-674.

Goslin, K., E. Birgbauer, G. Banker, and F. Solomon (1989) The role of cytoskeleton in organizing growth cones: A microfilament-associated growth cone component depends upon microtubules for its localization. J. Cell Biol. 109: 1621-1631.

Griffin, J. W., D. L. Price, D. B. Drachman, and J. Morris (1981) Incorporation of axonally transported glycoproteins into axolemma during nerve regeneration. J. Cell Biol. 88: 205-214.

Griffiths, G., and K. Simons (1986) The trans Golgi network: Sorting at the exit site of the Golgi complex. Science 234: 438-443.

Jacobson, R. D., I. Virag, and J. H. P. Skene (1986) A protein associated with axon growth, GAP-43, is widely distributed and developmentally regulated in rat CNS. J. Neurosci. $6: 1843-1855$

Johnson, G., M. Gloria, and C. Noghueira Araujo (1981) A simple method of reducing the fading of immunofluorescence microscopy. J. Immunol. Methods 43: 349-350.

Laemmli, U. K. (1970) Cleavage of structural proteins during the assembly of the head of bacteriophage T4. Nature 227: 680-685.

Levine, J., and M. Willard (1983) Redistribution of fodrin (a component of the cortical cytoplasm) accompanying capping of cell surface molecules. Proc. Natl. Acad. Sci. USA 80: 191-195.

Lindsley, T. A., P. DeCamilli, and G. A. Banker (1987) The influence of cell-cell contact on the distribution of synapsin I in hippocampal neurons in culture. Soc. Neurosci. Abstr. 13: 318.

Matus, A., R. Bernhardt, R. Bodmer, and D. Alaimo (1986) Microtubule-associated protein 2 and tubulin are differently distributed in the dendrites of developing neurons. Neuroscience 17:371-389.

Meiri, K. F., K. H. Pfenninger, and M. B. Willard (1986) Growthassociated protein, GAP-43, a polypeptide that is induced when neurons extend axons, is a component of growth cones and corresponds to pp46, a major polypeptide of a subcellular fraction enriched in growth cones. Proc. Natl. Acad. Sci. USA 83: 3537-3541.

Meiri, K. F., M. I. Johnson, and M. Willard (1988) Distribution and phosphorylation of the growth associated protein GAP-43 in regenerating sympathetic neurons in culture. J. Neurosci. 8: 2571-2581.

Nestler, E. J., and P. Greengard (1986) Synapsin 1: A review of its distribution and biological regulation. Prog. Brain Res. 69: 323-339.

Oestreicher, A. B., and W. H. Gispen (1986) Comparison of the immunocytochemical distribution of the phosphoprotein B-50 in the cerebellum and hippocampus of immature and adult rat brain. Brain Res. 375: 267-279.

Oestreicher, A. B., H. Zwiers, P. Schotman, and W. H. Gispen (1981) Immunohistochemical localization of a phosphoprotein (B-50) isolated from rat brain synaptosomal plasma membranes. Brain Res. Bull. 6: 145-153.

Pfenninger, K. H., and M. F. Maylie-Pfenninger (1981) Lectin labeling of sprouting neurons. II. Relative movement and appearance of glycoconjugates during plasmalemmal expansion. J. Cell Biol. 89: 547559.

Redshaw, J. D., and M. A. Bixby (1984) Proteins of fast axonal transport in the regenerating hypoglossal nerve of the rat. Can. J. Physiol. Pharmlacul. 62: 1387-1393.

Shaw, G., G. A. Banker, and K. Weber (1985) An immunofluorescence study of neurofilament protein expression by developing hippocampal neurons in tissue culture. Eur. J. Cell Biol. 3: 205-216.

Simons, K., and S. D. Fuller (1985) Cell surface polarity in epithelia Annu. Rev. Cell Biol. 1: 243-288. 
Skene, J. H. P. (1989) Axonal growth-associated proteins. Annu. Rev. Neurosci. 12: 127-156.

Skene, J. H. P., and I. Virag (1989) Post-translational membrane attachment and dynamic fatty acylation of a neuronal growth cone protein, GAP-43. J. Cell Biol. 108: 613-625.

Skene, J. H. P., and M. Willard (1981a) Changes in axonally transported proteins during axon regeneration in toad retinal ganglion cells. J. Cell Biol. 89: 86-96.

Skene, J. H. P., and M. Willard (1981b) Axonally transported proteins associated with axon growth in rabbit central and peripheral nervous systems. J. Cell Biol. 89: 96-103.

Skene, J. II. P., and M. Willard (1981c) Characteristics of growthassociated polypeptides in regenerating toad retinal ganglion cell axons. J. Neurosci. 1: 419-426.

Skene, J. H. P., R. D. Jacobson, G. J. Snipes, C. B. McGuire, J. J. Norden, and J. A. Freeman (1986) A protein induced during nerve growth (GAP-43) is a major component of growth-cone membranes. Science 233: 783-786.

Tartakoff, A. M., and P. Vassali (1983) Lectin-binding sites as markers of Golgi subcompartments: Proximal-to-distal maturation of oligosaccharides. J. Cell Biol. 97: 1243-1248.

VanHooff, C. O. M., J. C. M. Holthuis, A. B. Oestreicher, J. Boonstra, P. N. E. DeGraan, and W. H. Gispen (1989) Nerve growth factorinduced changes in the intracellular localization of the protein kinase $\mathrm{C}$ substrate B-50 in pheochromocytoma PC1 2 cells. J. Cell Biol. 108: $1115-1126$.

Virtanen, I., P. Ekblom, and P. Laurila (1980) Subcellular compartmentation of saccharide moieties in cultured neuronal and malignant cells. J. Cell Biol. 85: 429-434.

Yamada, K. M., B. S. Spooner, and N. K. Wessells (1971) Ultrastructure and function of growth cones and axons of cultured nerve cells. J. Cell Biol. 49: 614-635. 